\title{
Fomento de la lactancia materna en el municipio de Sabaneta
}

\author{
Promotion of breastfeeding in the municipality of Sabaneta
}

Promoção do aleitamento materno no município de sabaneta

Andrés Velásquez ${ }^{1}$, Nayibe Yepes', Catalina Fajardo', Isabel Orozco'

Fecha correspondencia:

Recibido: mayo 4 de 2016.

Revisado: junio 1 de 2016.

Aceptado: junio 20 de 2016.

Forma de citar:

Velásquez A, Yepes N, Fajardo C,

Orozco I. Fomento de la lactancia materna en el municipio de Sabaneta. Rev CES Salud Pública. 2016; 7 (2)

Open access

(c) Copyright

Licencia creative commons

Ética de publicaciones

Revisión por pares

Gestión por Open Journal System

ISSN: 2145-9932

Sobre los autores:

1. Médicos Internos, Fundación Universitaria San Martín, Sede

Sabaneta, Antioquia.

Comparte

\section{Resumen}

Objetivo: Evaluar la lactancia materna en madres pertenecientes al programa FAMI-Sabaneta en el 2014. Materiales y métodos: Estudio descriptivo, se realizó una encuesta a 49 madres del programa FAMI del municipio de Sabaneta sobre las características sociales, inicio, duración de la lactancia materna y las causas de destete durante el año 2014. Se determino la incidencia estadística de los factores asociados al abandono de la lactancia materna. Resultados: Se encontró un 39\% de madres adolescentes, el $84 \%$ de las madres tenían un nivel como máximo nivel educativo secundaria, no se dio cuenta de LME en un 10,2\%, hora y duración de la primera toma de contacto adecuada: $16 \%$, las causas del destete: el niño no le gustaba $(22,2 \%)$, no le gustaba a la madre, la edad del destete por personal de salud $(11,1 \%)$ cada variable. Conclusiones: El destete se asoció con datos objetivos más que con las percepciones maternas. Se requiere educación superior en salud personal amamantando y fortaleciendo el programa FAMILIA - Sabaneta.

Palabras clave: Lactancia materna, lactancia materna exclusiva, lactancia materna complementaria, destete, programa FAMI, abandono de la lactancia materna.

\section{Abstract}

Aim: Assess breastfeeding mothers linked to FAMI-Sabaneta program in 2014. Materials and methods: A descriptive study, a survey was conducted on 49 mothers of FAMI community program about social characteristics, onset, duration of breastfeeding and weaning causes during 2014 year. The incidences of leaving factors of breastfeeding were statistically determined. Results: Teenage Mothers: $39 \%$, secondary education: $84 \%$ did not realize LME: $10.2 \%$, time and duration of the first proper contact: $16 \%$, causes of weaning: the child did not like (22.2\%), did not like to mother, age of weaning by health personnel (11.1\% each variable). Conclusions: The weaning was associated with objective data rather than maternal perceptions. Higher education is required in personal health breastfeeding and strengthening the FAMI - Sabaneta program.

Keywords: Breastfeeding, exclusive breastfeeding, complementary breastfeeding, weaning, FAMI program, leaving breastfeeding. 


\section{Resumo}

Objetivo: Avaliar as mães que amamentam pertencentes ao programa FAMI-Sabaneta em 2014. Materiais e métodos: estudo descritivo, foi realizada uma pesquisa em 49 mães de programa FAMI do município de Sabaneta na dimensão social, início, duração do aleitamento materno e as causas de desmame durante 2014. a incidência estatística de fatores associados foram determinados ao abandono amamentação. Resultados: 39\% das mães adolescentes foi encontrado, 84\% das mães tinham ensino secundário nível máximo, não percebeu LME em 10,2\%, a hora ea duração do primeiro tomar contacto adequado: $16 \%$ as causas do desmame, a criança não gostava (22,2\%), ele não gostava da mãe, idade de desmame de saúde $(11,1 \%)$ cada variável. Conclusões: 0 desmame é associado com dados objetivos ao invés de percepção materna. 0 ensino superior é necessária em enfermagem de saúde pessoal e fortalecimento FAMÍLIA programa - Sabaneta.

Palavras-chave: Amamentação, aleitamento materno exclusivo, aleitamento complementar, o desmame, o programa FAMI, o abandono da amamentação.

\section{Introducción}

La lactancia materna un métodos importante, práctico, y económico en la alimentación infantil, es un aporte esencial al crecimiento y desarrollo del niño a nivel psicológico, cognitivo, pondoestatural, afectivo e inmunológico y fortalece relación madre-hijo (1).

La organización mundial de la salud (OMS) define (2):

Lactancia materna exclusiva: "alimentación del lactante con leche materna de la madre u otra mujer sin suplemento solido o liquido", durante los primeros seis meses.

Lactancia materna complementaria: "a partir del sexto mes además de amamantar se inicia introducción de otros alimentos".

Lactancia materna predomínate: “leche humana como principal fuente recibe otras bebidas.

Lactancia materna parcial: biberón con al menos un sucedáneo de la leche materna.

Lactancia materna simbólica: dada como consuelo, por periodos ocasionales de menos de 15 minutos al día.

Este proceso, se ve afectado por circunstancias que llevan a las madres a desfallecer en la práctica. En Colombia se reflejan las principales causas de abandono a nivel mundial: consumo de leche de fórmula, técnicas inadecuadas, alteraciones del pezón, dolor, creencia cultural de producción inefectiva y de necesidad de inicio temprano de otros alimentos y reingreso laboral temprano (3).

Colombia cuenta con el programa FAMI4 (familia-mujer-infancia) del Instituto Colombiano de Bienestar Familiar, creado en el año 2007 para brindar apoyo a la primera infancia. Este fomenta la lactancia materna con la ayuda de MADRES FAMI, mujeres líderes de la comunidad capacitadas, que trabajan con mujeres gestantes o con hijos menores de 2 años, en situación de vulnerabilidad psicoafectiva, nutricional, económica y social, del SISBEN 1 y 2. 
Partiendo del impacto positivo de la lactancia sobre el crecimiento y desarrollo del niño, además del fortalecimiento afectivo del binomio madre-hijo, y apoyados en este programa detectamos la incidencia de la lactancia materna y factores influyentes en su abandono en madres usuarias del programa FAMI-Sabaneta en el 2014; para tener información de esta población para una futura intervención que busque fomentar y fortalecer la lactancia materna.

\section{Materiales y métodos}

Tipo de estudio: Descriptivo. Población: Madres FAMI del municipio de Sabaneta. Tamaño de muestra: 49 mujeres con niños menores de un año que pertenecen al programa FAM-Sabaneta.

Técnica de recolección de la información: Encuesta ajustada a la realzada por Marco Sosa en el año 1999 (5); contiene preguntas acerca de lactancia materna, duración, alimentación complementaria, experiencias durante el amamantamiento y motivo de abandono.

Unidad de análisis: Cada madre usuaria del programa FAMI-Sabaneta en el 2014.

\section{Resultados}

En total se entrevistaron 49 madres entre los 16 y 35 años.

El nivel educativo del $84 \%$ de las madres es secundaria (tabla 1).

Tabla 1. Nivel educativo de las madres

\begin{tabular}{lcc}
\hline \multicolumn{1}{r}{ Nivel educativo } & No & \% \\
\hline Primaria completa & 4 & $8 \%$ \\
Primaria incompleta & 4 & $8 \%$ \\
Secundaria completa & 16 & $33 \%$ \\
Secundaria incompleta & 17 & $35 \%$ \\
Superior completa & 6 & $12 \%$ \\
Superior incompleta & 2 & $4 \%$ \\
Total general & 49 & $100 \%$ \\
\hline
\end{tabular}

Al $64 \%$ de las madres recibieron el hijo inmediatamente, de estas solo 1:4 permanecieron con su hijo más de 15 minutos. Además de las $25 \%$ que no recibieron su hijo de inmediato casi la mitad permanecieron con su hijo menos de quince minutos. (Tabla 2)

El 18\% de las entrevistadas no iniciaron amamantamiento a la primera hora (figura 1), momento crítico para asegurar una producción de leche y adherencia a la lactancia.

De las 49 madres $51 \%$ no amamantaban en la actualidad, una no inició, 6 solo dieron lactancia materna exclusiva, 3 amamantaron hasta los 18 meses y 1 de cada 5 mujeres no cumplen con la lactancia materna exclusiva (figura 2). 
Tabla 2. Tiempo de contacto vs momento de entrega del hijo a la madre.

\begin{tabular}{llccc}
\hline & & \multicolumn{3}{c}{ Tiempo de contacto } \\
\cline { 2 - 5 } & \multicolumn{1}{c}{$\begin{array}{c}\text { Mayor de 15 } \\
\text { minutos }\end{array}$} & $\begin{array}{c}\text { Menor de 15 } \\
\text { minutos }\end{array}$ & Total \\
\cline { 2 - 5 } $\begin{array}{l}\text { Momento de } \\
\text { entrega }\end{array}$ & Inmediato & 8 & 24 & 32 \\
\cline { 2 - 5 } & No inmediato & 10 & 7 & 17 \\
\cline { 2 - 5 } & Total & 18 & 31 & 49 \\
\hline
\end{tabular}

\section{N: 49}

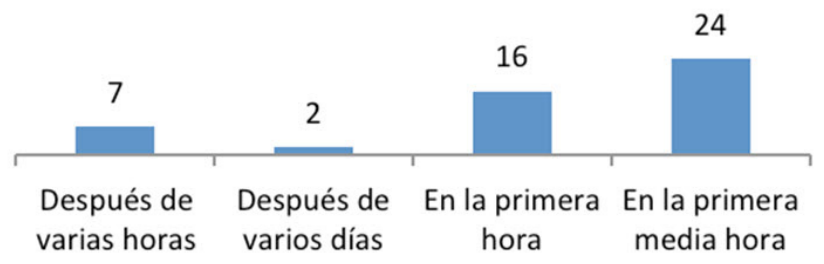

Figura 1. Periodo de tiempo en el que se alimento con leche materna

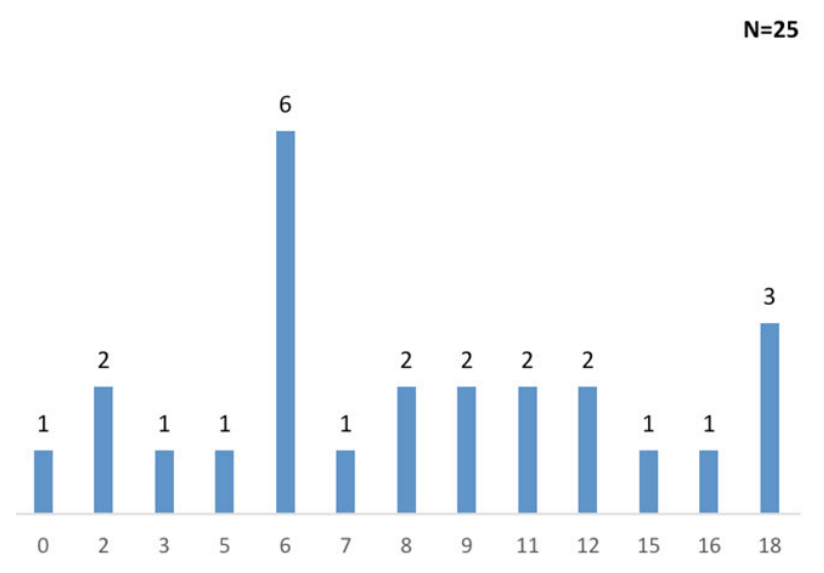

Figura 2. Mujeres que no amamantan vs madre usuaria del programa

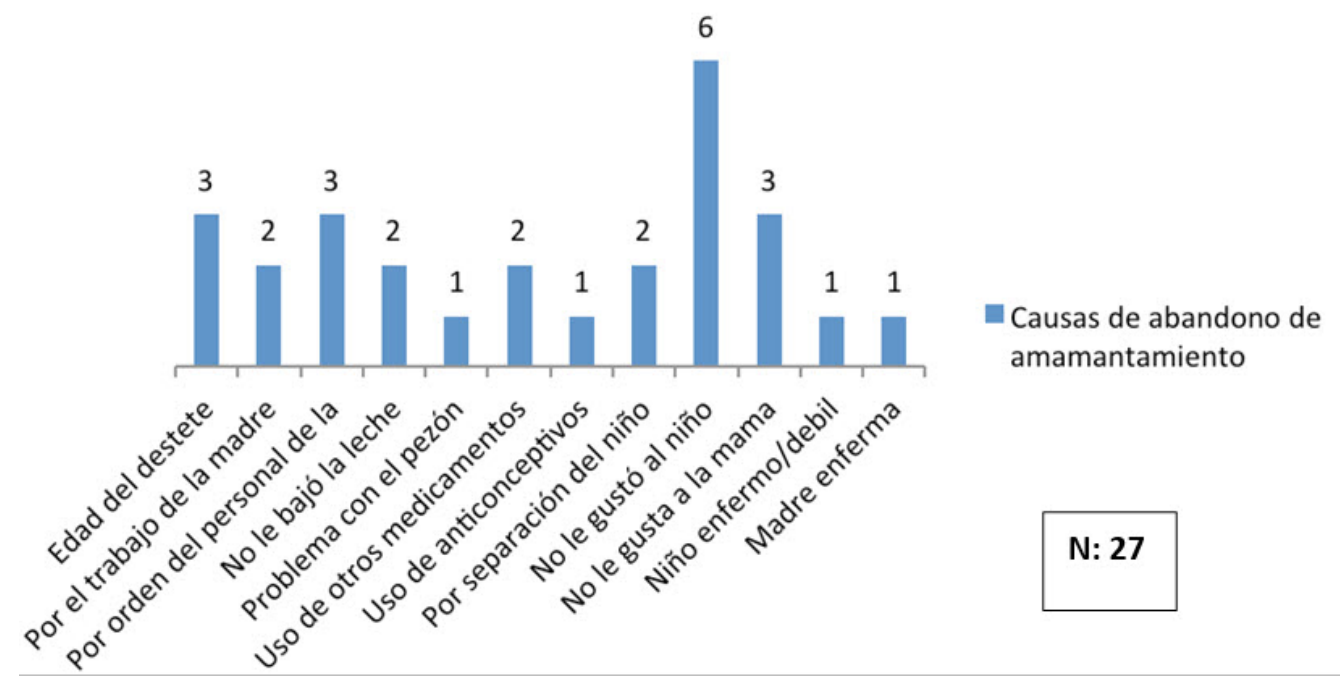

Figura 3. Causas de abandono de amamantamiento 
De 27 madres que abandonaron el amamantamiento las causa más representativas fueron: no le gusto al niño (6 madres), por edad del destete, por orden del personal de la salud y no le gustó a la mamá (3 madres en cada variable). (Figura 3)

\section{Discusión}

Este estudio mostro que 1:6 mujeres del programa FAMI-Sabaneta de ICBF no realizan lactancia materna exclusiva (6 meses), cifra aún desalentadora a pesar de esta por encima de la cifra de la Encuesta Nacional de Salud 2010 (ENDS) (으) y la Encuesta Nacional de Situación Nutricional en Colombia 2010 (ENSIN) (7), este aumento demuestra el aporte positivo del programa FAMI, a la mejora la lactancia exclusiva.

En la población en cuestión el bajo nivel educativo limitan los procesos educativos para la salud por lo que requiere mayor acompañamiento en la práctica de la lactancia.

Se observó aunque solo 1:6 casos tuvieron entrega inmediata del niño a la madre después de nacimiento y una duración de primer contacto por más de 15 minutos, tiempos que según las OMS aportan a garantizar la lactancia, el 82\% de las madres iniciaron la lactancia tempranamente, en la primera hora, lo que asegura mejor producción y adherencia al amamantamiento; cifra muy por encima del $48.9 \%$, promedio Nacional. Mostrando el beneficio de implementar el programa FAMI de Bienestar Familiar.

Comparando las causas del destete con Envigado (5) población con características similares, se encontró que las principales causas fueron muy diferentes. Envigado: no le bajo leche $(46,7 \%)$, no le gusto al niño $(12,9 \%)$, edad del destete y por trabajo de la madre (7,6\%)6. Sabaneta: no le gusto al niño (22.2\%), no le gusto a la madre, edad del destete, orden de personal de la salud (11.1\% a cada variable). Detectamos que el destete generalmente se relaciona con percepciones maternas más que con datos objetivos; situación modificable y puede ser abarcada desde las madres lideres FAMI por lo que se considera que debe haber fortalecimiento de este programa mejorando así la calidad y tiempo de acompañamiento a las madres usuarias. Con esta investigación se ha aportado datos de interés para proponer y fortalecer, futuras intervenciones para mejorar la lactancia materna en Sabaneta con acciones coordinadas entre el equipo de salud y el programa FAMI-Sabaneta.

\section{Bibliografía}

1. Unicef. Lactancia materna. [Sitio de internet]. Disponible en: http://www.unicef. org.co/pdf/nutri4 pg55-126.pdf Pag 5

2. OMS. Lactancia materna. [Sitio de internet]. Disponible en: http://www.who.int/ topics/breastfeeding/es/

3. Profamilia. Lactancia materna y estado nutricional. [Sitio de internet]. Disponible en: http://www.profamilia.org.co/encuestas/Profamilia/Profamilia/images/stories/PDF-capitulos/Capitulo-11.pdf Pag 7

4. Hogar comunitario - Bienestar Familiar. FAMI. [Sitio de internet]. Disponible en: http://www.pedagogia-gensini-gavito.com.es/index.php?option=com content\&view=article\&id=113:hogar-comunitario-fami-bienestar-familiar\&cati$\underline{\mathrm{d}=130: \text { red-vinculaciones\&ltemid }=152}$ 
5. Alcaldía de Envigado. Evaluación y monitoreo de la lactancia materna en menores de un año del municipio de Envigado año 2009. [Sitio de internet]. Disponible en: http://www.envigado.gov.co/Secretarias/SecretariadeSalud/documentos/ Seguridad\%20Alimentaria/Investigacion $\% 20$ sobre\%20lactancia $\% 20$ materna\%20ano\%202009.pdf

6. Profamilia. Encuesta Nacional de Salud 2010 (ENDS). [Internet]. [Citado $2014 \mathrm{Ag}$ 4]. Disponible en http://www.profamilia.org.co/encuestas/Profamilia/Profami-

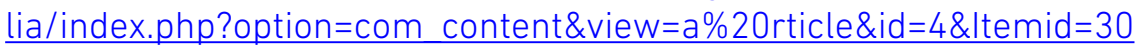

7. Colombia. Instituto Colombiano de Bienestar Familiar. Encuesta Nacional de Situación Nutricional en Colombia ENSIN 2010. [Internet]. [Citado 2014 Ag 4]. Disponible en http://www.icbf.gov.co/portal/page/portal/PortallCBF/NormatividadC/ENSIN1/ENSIN2010/LibroENSIN2010.pdf 